University of Nebraska - Lincoln

DigitalCommons@University of Nebraska - Lincoln

$11-1991$

\title{
Pyro-electro-optic phase gratings
}

Stephen Ducharme

University of Nebraska-Lincoln, sducharme1@unl.edu

Follow this and additional works at: https://digitalcommons.unl.edu/physicsducharme

Part of the Physics Commons

Ducharme, Stephen, "Pyro-electro-optic phase gratings" (1991). Stephen Ducharme Publications. 80.

https://digitalcommons.unl.edu/physicsducharme/80

This Article is brought to you for free and open access by the Research Papers in Physics and Astronomy at DigitalCommons@University of Nebraska - Lincoln. It has been accepted for inclusion in Stephen Ducharme Publications by an authorized administrator of DigitalCommons@University of Nebraska - Lincoln. 


\title{
Pyro-electro-optic phase gratings
}

\author{
Stephen Ducharme
}

Department of Physics and Astronomy, Center for Materials Research and Analysis, University of Nebraska, Lincoln, Nebraska 68588-0111

Received June 27, 1991

\begin{abstract}
A new physical mechanism is proposed for generating transient phase gratings in transparent pyroelectric materials. The mechanism combines the pyroelectric and electro-optic effects to convert spatial intensity variations into transient phase gratings. The grating diffraction efficiency increases with a figure of merit that is proportional to the pyroelectric, electro-optic, and absorption coefficients and is inversely proportional to the specific heat. The grating response time is proportional to the specific heat and inversely proportional to the thermal conductivity. Diffraction efficiencies that approach unity are predicted in several readily available electro-optic materials with modest pulse energies from $Q$-switched Nd:YAG lasers. Observation with cw laser sources is also proposed. The pyro-electro-optic mechanism is compared with other known mechanisms.
\end{abstract}

There are many mechanisms for generating transient optical phase gratings. Although particular advantage can be taken of semipermanent mechanisms such as the photorefractive and photochromic effects, there are also uses for purely transient mechanisms such as those based on the third-order nonlinear susceptibility, population change, or thermal $(\mathrm{d} n / \mathrm{d} T)$ mechanisms. Transient grating mechanisms permit the real-time interaction of interfering waves as in phase conjugation, image amplification and manipulation, optical modulation and switching, and other applications. I propose a new mechanism of transient optical phase grating formation, based on a combination of the pyroelectric $^{1}$ and electro-optic ${ }^{2}$ effects, called the pyroelectro-optic (PEO) effect.

A novel combination optical-to-optical modulator based on the pyroelectric and electro-optic effects was proposed and demonstrated in 1987 by Kalman and Toth. ${ }^{3}$ This device consists of a pyroelectric crystal connected in parallel to an electro-optic crystal. Light absorbed by the pyroelectric crystal produces a voltage that is applied to the electro-optic crystal. The refractive indices of the second crystal change owing to the electro-optic effect, thus altering the phase and/or the polarization of a probe beam that is passing through the electro-optic crystal. Since any pyroelectric material is electro-optic as well, both the input and probe beams may pass through the same crystal, as noted in Ref. 3. The proposed PEO mechanism builds on this by interfering two mutually coherent optical beams in a pyroelectric material to generate a transient phase grating, through the pyroelectric and electro-optic effects, which will then diffract an optical beam.

PEO gratings are formed by transient holography and can, without loss of generality, be treated in the plane-wave approximation; an image-bearing or Gaussian profile laser beam can be treated as a superposition of plane waves. Two mutually coherent linearly polarized plane-wave writing beams of vacuum wavelength $\lambda$ with wave vectors $\mathbf{k}_{1}$ and $\mathbf{k}_{2}$ and amplitudes $\mathbf{E}_{1}$ and $\mathbf{E}_{2}$ intersect in a pyroelectric material at an angle 2 $\theta$, beginning at time $t=0$ (see Fig. 1). The intensity of the interference pattern formed by the two beams is $I=I_{0}[1+\delta \cos (k z)]$, where $I_{0}=I_{1}+I_{2}$ is the total intensity, $\delta=$ $n c \epsilon_{0}\left|\mathbf{E}_{1} \cdot \mathbf{E}_{2}\right| /\left(I_{1}+I_{2}\right)$ is the intensity modulation, $I_{1}=n c \epsilon_{0}\left|\mathbf{E}_{1}\right|^{2} / 2$ is the intensity of beam 1 , and $\mathbf{k}_{g}=\mathbf{k}_{1}-\mathbf{k}_{2}$ is the grating wave vector. Some of the light is absorbed by the material, and the temperature $T$ rises according to the one-dimensional heat-transport equation (assuming, for simplicity, that the medium is thermally isotropic),

$$
\tilde{c} \frac{\partial T}{\partial t}=\kappa \frac{\partial^{2} T}{\partial z^{2}}+\alpha I-K\left(T-T_{0}\right),
$$

where $\tilde{c}$ is the heat capacity per unit volume, $\kappa$ is the thermal conductivity, $\alpha$ is the absorption coefficient, and $K\left(T-T_{0}\right)$ is the rate of heat loss out of the illuminated region into its surroundings at temperature $T_{0}$. The temperature in the material is the solution of Eq. (1) with the sinusoidal intensity variation and boundary condition $T(t=0)=T_{0}$. Then (with $K \ll \kappa k^{2}$ )

$$
\begin{aligned}
T= & T_{0}+\frac{\alpha I_{0}}{K}[1-\exp (-\gamma t)] \\
& +\delta \frac{\alpha I_{0}}{\kappa k_{g}{ }^{2}}[1-\exp (-\Gamma t)] \cos \left(k_{g} z\right),
\end{aligned}
$$

where $\Gamma=\kappa k_{g}{ }^{2} / \tilde{c}$ is the inverse of the grating rise time and $\gamma=K / \tilde{c}$ is the rate of increase of the average temperature of the illuminated region.

There will be a change in the electric polarization proportional to the change in temperature by the pyroelectric effect ${ }^{1} \Delta P_{j}=p_{j} \Delta T$, and consequently there will be an electric field $E_{j}=-\Delta P_{j} / \epsilon_{0}=$ $-p_{j} \Delta T / \epsilon_{0}$, where $p_{j}$ is the pyroelectric coefficient at constant stress and $\epsilon_{0}$ is the permittivity of free space. The index of refraction is altered through the linear electro-optic (Pockels) effect ${ }^{2} \Delta\left(1 / n^{2}\right)_{i}=$ $r_{i j} E_{j}$, where $r_{i j}$ is the linear electro-optic tensor and $\Delta\left(1 / n^{2}\right)_{i}$ is the change in the refractive-index ellipsoid. We consider here only the diagonal terms 


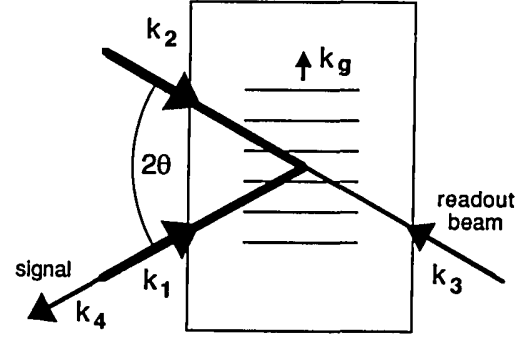

Fig. 1. Diagram of PEO grating formation and readout (for simplicity, refraction at the sample interfaces is not shown). Two mutually coherent beams $\mathbf{k}_{1}$ and $\mathbf{k}_{2}$ intersect at an angle $2 \theta$ in the material and form a phase grating with wave vector $\mathbf{k}_{g}$. A third beam $\mathbf{k}_{3}$ counterpropagating with beam $\mathbf{k}_{2}$ diffracts from the grating to form the signal beam $\mathbf{k}_{4}$. The grating planes (horizontal stripes) are also shown.

$i=1,2,3$ ( $i$ may take on the values $1-6$ in general), which correspond to changes in the lengths of the principle of the index ellipsoid. In this case the change in the $i$ th principle refractive index is $\Delta n_{i}=$ $-n_{i}{ }^{3} r_{i j} E_{j} / 2$, where $i$ is the direction of polarization of the probing light. The amplitude of the spatially varying part of the index of refraction in the material is then [with Eq. (2)]

$$
\Delta n_{i}=\delta \frac{F_{i} I_{0}}{\Gamma}[1-\exp (-\Gamma t)],
$$

where the PEO figure of merit of the medium is

$$
F_{i}=\frac{\alpha_{i} n_{i}{ }^{3} r_{i j} p_{j}}{2 \tilde{c} \epsilon_{0}}
$$

The figure of merit $F_{i}$ is intrinsic to the material and depends on the temperature and on the wavelengths and polarizations of the beams.

The refractive-index variation $\Delta n_{i}$ in Eq. (3) is a phase grating, or hologram, that has Bragg diffraction efficiency $\eta_{i}=\sin ^{2}\left(\pi L u_{i} \Delta n_{i} / \lambda \cos \theta\right)$, where $u_{i}=1$ for $s$-polarized readout, $u_{i}=\cos 2 \theta$ for $p$ polarized readout, and $L$ is the interaction length. ${ }^{4}$ The PEO diffraction efficiency is therefore

$$
\eta_{i}=\sin ^{2}\left\{\frac{\pi L u_{i}}{\lambda \cos \theta} \frac{\delta F_{i} I_{0}}{\Gamma}[1-\exp (-\Gamma t)]\right\} .
$$

The diffraction efficiency builds steadily once illumination begins until the temperature modulation approaches a steady state after several time constants of $1 / \Gamma$. When the grating is formed by a short pulse $(\Gamma \tau \ll 1)$, the diffraction efficiency increases quadratically in time and reaches $\eta_{i}=$ $\sin ^{2}\left(\pi L u_{i} \delta F_{i} W_{0} / \lambda \cos \theta\right)$ by the end of the pulse, depending on the pulse energy density $W_{0} \approx I_{0} \tau$, followed by a decay with a time constant $1 / \Gamma$ after the pulse ends. When the grating is formed by a long pulse or cw illumination of duration $\tau \gg 1 / \Gamma$, the diffraction efficiency quickly reaches its steadystate value $\eta_{i}=\sin ^{2}\left(\pi L u_{i} \delta F_{i} I_{0} / \lambda \Gamma \cos \theta\right)$.

Table 1 displays the figures of merit for a selection of electro-optic crystals ranging from ferroelectrics to semiconductors to organic crystals. This selection is necessarily limited not only in the selection of materials (e.g., electro-optic polymers could also be included) but also in the range of temperatures and wavelengths over which the indicated properties may change considerably, as demonstrated by the two entries for $\mathrm{BaTiO}_{3}$. The exceptionally large figure of merit $F_{3}$ in the second $\mathrm{BaTiO}_{3}$ entry occurs just $20^{\circ} \mathrm{C}$ below the Curie temperature and at a wavelength near the absorption edge where the electro-optic and pyroelectric constants and the index of refraction all have particularly large values. The temperature and wavelength are important parameters considering that most of the values in Table 1 were measured at temperatures far from the Curie temperature (for ferroelectrics) and at wavelengths far from the absorption edge, and therefore the figures of merit are not optimum.

The holographic sensitivity can be defined as the index change per unit energy density. If we assume illumination of finite duration $\tau$ and unity modulation $\delta=1$, the PEO sensitivity is

$$
S_{i}=\frac{\Delta n_{i}}{I_{0} \tau}=\frac{F_{i}}{\Gamma \tau}[1-\exp (-\Gamma \tau)]
$$

The sensitivity drops to $50 \%$ for a pulse length of $\tau=1.6 / \Gamma$, while the diffraction efficiency reaches at least $63 \%$ of its saturated value in the same time. A pulse length of $\tau \approx 1.6 / \Gamma$ represents a reasonable compromise between sensitivity and diffraction efficiency; shorter pulses use the pulse energy more efficiently, but longer pulses achieve higher diffraction efficiency. Note, though, that at high intensity $100 \%$ diffraction efficiency can be achieved when $\Gamma \tau \ll 1$ (and $S_{i}=F_{i}$ ), but the diffraction efficiency begins to fall after reaching $100 \%$ owing to the sinusoidal dependence in Eq. (5). At even higher intensities, the diffraction efficiency will oscillate until $\Gamma t \gg 1$.

The PEO mechanism differs functionally from the photorefractive mechanism in several ways. The photorefractive effect produces internal electric fields through drift and diffusion of photogenerated carriers, which is different from the thermal origin of the PEO electric fields. One functional distinction between photorefractive and PEO gratings is that $\mathrm{PEO}$ gratings are transient, decaying with time constant $1 / \Gamma$ (which is typically less than $10^{-3} \mathrm{~s}$ ) after illumination has ceased, whereas photorefractive gratings persist long after illumination has ceased. Another distinction is that the PEO diffraction efficiency is independent of the orientation of the grating planes while the direction of the photorefractive electric field is (usually) along the grating wave vector, thus the photorefractive diffraction efficiency is highly dependent on grating orientation through the anisotropy of the electro-optic effect.

PEO gratings will always be accompanied by a thermal grating; the temperature variation of Eq. (2) produces a corresponding variation in the index of refraction $\Delta n_{i}=\zeta_{i} \Delta T$, where $\zeta_{i}=\mathrm{d} n_{i} / \mathrm{d} T$. The diffraction efficiency of the thermal grating has the same form as Eq. (5) with a figure of merit $F_{T i}=\alpha_{i^{\prime}} \zeta_{i} / \tilde{c}$. The ratio between the thermal and PEO figures of merit is $F_{i} / F_{T i}=n_{i}{ }^{3} r_{i j} p_{j} / 2 \zeta_{i} \epsilon_{0}$. For example, the ratio $F_{3} / F_{T 3}=114$ for $\mathrm{BaTiO}_{3}$ with 
Table 1. Material Properties and Derived PEO Electro-Optic Figures of Merit $F_{i}$ for Representative Electro-Optic Crystals ${ }^{a}$

\begin{tabular}{lccccccc}
\hline & $i j$ & $n_{i}{ }^{3} r_{i j}(\mathrm{pm} / \mathrm{V})$ & $p_{j}\left(\mu \mathrm{C} / \mathrm{m}^{2} \mathrm{~K}\right)$ & $\tilde{c}\left(\mathrm{~J} / \mathrm{cm}^{3} \mathrm{~K}\right)$ & $\kappa(\mathrm{W} / \mathrm{mK})$ & $F_{i}\left(10^{-3} \mathrm{~cm}^{2} / \mathrm{J}\right)$ & $\Gamma\left(10^{6} / \mathrm{s}\right)$ \\
\hline $\mathrm{BaTiO}_{3}{ }^{b}$ & 33 & 1376 & 280 & 2.60 & 5.8 & 4.2 & 0.56 \\
$\mathrm{BaTiO}_{3}{ }^{c}$ & 33 & 6401 & 1200 & 3.97 & 8.0 & 87.4 & 0.54 \\
$\mathrm{KNbO}_{3}$ & 33 & 710 & 100 & 3.00 & 5.0 & 0.7 & 0.35 \\
$\mathrm{LiNbO}_{3}$ & 33 & 357 & 83 & 2.88 & 5.6 & 0.3 & 0.42 \\
$\mathrm{SBN}^{6} 60^{b}$ & 33 & 2859 & 850 & 3.00 & 6.0 & 22.9 & 0.45 \\
$\mathrm{CdS}^{d}$ & 33 & 51 & 3.5 & 0.11 & 21.0 & 0.05 & 46 \\
$\mathrm{CdSe}^{d}$ & 33 & 59 & 4.0 & 0.09 & 9.0 & 0.08 & 26 \\
$\mathrm{MNA}^{e}$ & 11 & 536 & 2.0 & 0.62 & 1.0 & 0.05 & 0.27 \\
$\mathrm{MNMA}^{f}$ & 33 & 111 & 12.0 & 0.60 & 1.0 & 0.06 & 0.41 \\
\hline
\end{tabular}

${ }^{a}$ Material properties are from Ref. 5 unless otherwise noted. Unless otherwise noted, $\alpha=0.5 \mathrm{~cm}^{-1}$, $2 \theta=1^{\circ}, \lambda=632.8 \mathrm{~nm}$, and $T \approx 24^{\circ} \mathrm{C}$.

${ }^{b}$ At $\lambda=514.5 \mathrm{~nm}, n_{3}{ }^{3} r_{33}$ from Ref. 6.

${ }^{\mathrm{C} A t} T=110^{\circ} \mathrm{C}$ and $\lambda=442 \mathrm{~nm}$.

${ }^{d} \tilde{c}$ from Ref. 7 and $\kappa$ from Ref. $8, \lambda=1064 \mathrm{~nm}$.

${ }^{e}$ Molecular crystal 2-methyl-4-nitroaniline, $n_{1}{ }^{3} r_{11}$ from Ref. 9.

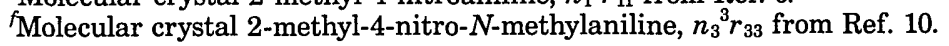

optical polarization along the polar axis $(i=3)$. The primary distinction between thermal and PEO gratings is the dependence on polarization; their temporal responses are identical. While both mechanisms exhibit a small amount of birefringence through $n_{i}$, the PEO mechanism has the additional dependence of the electro-optic coefficients $r_{i j}$ so that the PEO grating is usually highly anisotropic while that of the thermal grating is usually not.

Another common mechanism for producing transient phase gratings is through the third-order nonlinear susceptibility, ${ }^{11}$ or the nonlinear index of refraction $\Delta n=N_{2} I$. The highly nonlinear material polyacetylene has a nonresonant nonlinear index $N_{2} \approx 10^{-12} \mathrm{~cm}^{2} / \mathrm{W}$, much lower than the equivalent nonlinear index $F_{i} / \Gamma \geqslant 10^{-9} \mathrm{~cm}^{2} / \mathrm{W}$ for the first five PEO materials listed in Table 1.

Consider a PEO grating formed by 10 -ns-long pulses of equal energy density from the $\lambda=532 \mathrm{~nm}$ second-harmonic output of a Nd:YAG laser intersecting at the angle of $2 \theta=1^{\circ}$ in a 5 -mm-thick crystal of $\mathrm{BaTiO}_{3}$. The thermal relaxation time $1 / \Gamma=\tilde{c} / \kappa k_{g}^{2}=2.8 \mu \mathrm{s}$ is much longer than the laser pulse length, and the PEO figure of merit $F_{3}=$ $4.2 \times 10^{-3} \mathrm{~cm}^{2} / \mathrm{J}$ from Table 1 . From Eq. (5), in the short-pulse limit, an energy density of $5 \mathrm{~mJ} / \mathrm{cm}^{2}$ (with $\delta=1$ ) yields a diffraction efficiency of $34 \%$. A diffraction efficiency of $100 \%$ is attained with an energy density of $8.2 \mathrm{~mJ} / \mathrm{cm}^{2}$.

Consider now a cw source such as an argon-ion laser operating at $514.5 \mathrm{~nm}$ that forms a PEO grating in $\mathrm{BaTiO}_{3}$ with the following conditions: $\delta=1$, $L=5 \mathrm{~mm}, 2 \theta=1^{\circ}(1 / \Gamma=3.6 \mu \mathrm{s})$, and $I_{0}=$ $100 \mathrm{~W} / \mathrm{cm}^{2}$. The expected steady-state diffraction efficiency from Eq. (5) is $\eta=0.2 \%$. A cw PEO measurement in a photorefractive crystal such as $\mathrm{KNbO}_{3}$ or $\mathrm{BaTiO}_{3}$ will be overwhelmed by the much larger photorefractive grating, unless care is taken to align the grating wave vector $\mathbf{k}_{g}$ perpendicular to the crystal $c$ axis and the polarization of the read and signal beams primarily along the $c$ axis.

The pyroelectric and electro-optic effects may be combined to advantage in a new mechanism for generating transient phase gratings. The PEO grating diffraction efficiency is intensity dependent, and the response time is intensity independent, similar to thermal and nonlinear index mechanisms but in contrast with the photorefractive mechanism. In nonresonant situations (i.e., away from an absorption feature) the PEO mechanism has a higher sensitivity than thermal or nonlinear index mechanisms but a lower sensitivity than photorefractive mechanisms. The inherently transient nature of PEO gratings provides both opportunity, as in dynamic image processing, computing, and communications, where rapid response is desired, and limitation, as in data storage or in optical neural networks, where latency is important.

I thank W. E. Moerner for helpful discussions. Support is acknowledged from the Center for Materials Research and Analysis through the Nebraska Research Initiative, the U.S. Office of Naval Research (through IBM Corporation), and the California Department of Employment Development.

\section{References}

1. P. Nye, Physical Properties of Crystals (Oxford U. Press, London, 1967).

2. A. Yariv, Quantum Electronics, 3rd ed. (Wiley, New York, 1989).

3. P. Kalman and A. Toth, Ferroelectrics 75, 173 (1987).

4. H. Kogelnik, Bell Syst. Tech. J. 48, 2909 (1969).

5. K.-H. Hellwege and A. M. Hellwege, LandoltBörnstein: Numerical Data and Functional Relationships in Science and Technology, New Series, Vol. 3, No. 11 (Springer-Verlag, Berlin, 1979).

6. S. Ducharme and J. Feinberg, IEEE J. Quantum Electron. QE-23, 2116 (1987).

7. Y. S. Touloukian and E. H. Buyco, Specific Heat: Nonmetallic Solids (Plenum, New York, 1970).

8. C. M. Bhandari and D. M. Rowe, Thermal Conduction in Semiconductors (Wiley, New York, 1988).

9. G. F. Lipscomb, A. F. Garito, and R. S. Narang, Appl. Phys. Lett. 38, 663 (1981).

10. K. Sutter, C. Bosshard, M. Ehrensperger, P. Günter, and R. J. Twieg, IEEE J. Quantum Electron. 24, 2362 (1988).

11. R. W. Hellwarth, J. Opt. Soc. Am. 67, 1 (1977). 


\title{
Pyro-electro-optic phase gratings: erratum
}

\section{Stephen Ducharme}

Department of Physics and Astronomy, Center for Materials Research and Analysis, University of Nebraska, Lincoln, Nebraska 68588-0111

\begin{abstract}
My recent Letter ${ }^{1}$ omitted an assumption that restricts the range of validity of the results given. The relation $E_{j}=-\Delta P_{j} / \epsilon_{0}$ in the paragraph after Eq. (2) is valid only when the grating wave vector $\mathbf{k}_{g}$ is parallel to the direction $j$ of the pyroelectric polarization. The spatially modulated part of the pyroelectric field $\mathbf{E}$ will in general be parallel to the grating wave vector $\mathbf{k}_{g}$ because $\boldsymbol{\nabla} \times \mathbf{E}=0$. The sentence beginning "Another distinction..." at the end of the last full paragraph on p. 1792 is consequently inapplicable. I thank Marko Zgonik for pointing out this omission to me.
\end{abstract}

\section{Reference}

1. S. Ducharme, Opt. Lett. 16, 1791 (1991). 